\title{
A Comment on the Chaotic Behaviour of van der Pol Equations with an External Periodic Excitation
}

\author{
W.-H. Steeb \\ Department of Applied Mathematics and Nonlinear Studies, Rand Afrikaans University, \\ PO Box 524, Johannesburg 2000, South Africa \\ Jeun Chyuan Huang \\ Institute of Electronics National Chiao-Tung University Hsinchu, Taiwan, ROC \\ Yih Shun Gou \\ Department of Electrophysics, National Chiao-Tung University, Hsinchu, Taiwan, ROC \\ Z. Naturforsch. 44a, 160-162 (1989); received November 8, 1988 \\ The limit cycle system with an external periodic force $\mathrm{d}^{2} u / \mathrm{d} t^{2}-a\left(1-u^{2}\right) \mathrm{d} u / \mathrm{d} t+u^{n}=k \cos (\Omega t)$ \\ $(n=1,3,5, \ldots)$ can show chaotic behaviour for certain values of a, $\mathrm{k}$ and $\Omega$. We study the influence \\ of $n$ on the chaotic behaviour. For $n=1$ we select values which result in chaotic motion of the system. \\ Then we investigate the behaviour of the system for $n=3,5$ and 7 . Introducing the nonlinearity \\ $u^{n}(n=3,5,7)$ gives the surprising result that the chaotic motion ceases to exist.
}

It is well known that the differential equation

$$
\frac{\mathrm{d}^{2} u}{\mathrm{~d} t^{2}}+f(u) \frac{\mathrm{d} u}{\mathrm{~d} t}+g(u)=0
$$

shows (stable) limit cycle behaviour if the functions $f$ and $g$ satisfy the following conditions [1]:

i) $f$ is even; $g$ is odd, both are continuous for all $u$, and $f(0)<0$;

ii) $u g(u)>0$ for $u \neq 0$;

iii) $g$ is Lipschitzian;

iv) $F(u) \rightarrow \pm \infty$ as $u \rightarrow \pm \infty$, where

$$
F(u)=\int_{0}^{u} f(s) \mathrm{d} s
$$

v) $F$ has a single positive zero at $u=a$ and is monotone increasing for $u \geq a$. The equation

$$
\frac{\mathrm{d}^{2} u}{\mathrm{~d} t^{2}}-a\left(1-u^{2}\right) \frac{\mathrm{d} u}{\mathrm{~d} t}+u^{n}=0
$$

with $n$ odd and $a>0$ satisfies these conditions; i. e., we find stable limit cycle behaviour. For $n=1$ we obtain the famous van der Pol equation [2]. The cases with $n=3,5, \ldots$ are sometimes called generalized van der Pol equations.

Reprint requests to Prof. W.-H. Steeb, Department of Applied Mathematics, Rand Africaans University, PO Box 524, Johannesburg 2000, South Africa.
In the present note we study (2) with an external periodic force, i.e.,

$$
\frac{\mathrm{d}^{2} u}{\mathrm{~d} t^{2}}-a\left(1-u^{2}\right) \frac{\mathrm{d} u}{\mathrm{~d} t}+u^{n}=k \cos (\Omega t) .
$$

We are in particular interested in the chaotic behaviour in dependence on $n$ and $a$. The chaotic behaviour is characterized by the maximal one dimensional Lyapunov exponent. To find the maximal onedimensional Lyapunov exponent, system (3) together with its variational equation is integrated.

Parlitz and Lauterborn [3] found chaotic behaviour for the case $n=1$. For example, $a=5, k=5$, and $\Omega=2.466$ lead to chaotic motion. For $n=3$ and $n=5$ chaotic behaviour has been found by Ueda and Akamatsu [4] and Steeb and Kunick [5]. For example, if $n=3$ one finds chaotic motion for $a=0.2, k=17$, and $\Omega=4$. The chaotic bahaviour of the van der Pol equation with an external periodic force is restricted to large $a$, for example $a=5$. For small $a$ no chaotic motion exists. This is obvious since for small values of $a$ the stable limit cycle of the van der Pol equation is approximately given by $u(t)=2 \sin t$, i.e., the limit cycle is nearly a circle. On the other hand, for $n=3$ chaotic motion appears for small values of $a$ and appropriate values of $k$ and $\Omega$. Then the nonlinear term $u^{3}$ is responsible for the chaotic behaviour. It is known that the equation $\ddot{u}+u^{3}=k \cos (\Omega t)$ can show chaotic motion. 


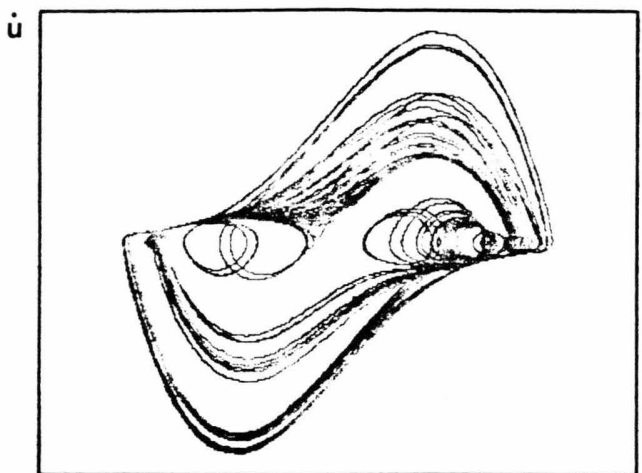

u

Fig. 1. Phase portrait of Van der Pol equation with external periodic force where $a=5, k=5, \Omega=2.466$, and $n=1$ (100 cycles).

$P(\omega)$

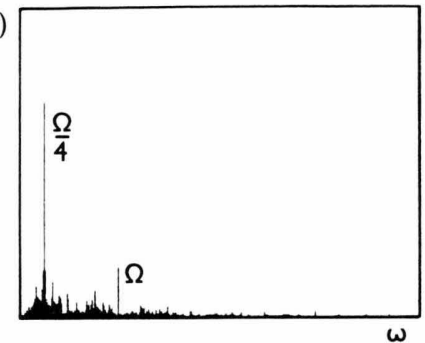

Fig. 3. Power spectrum of Van der Pol equation with external periodic force where $a=5, k=5, \Omega=2.466$, and $n=1$.

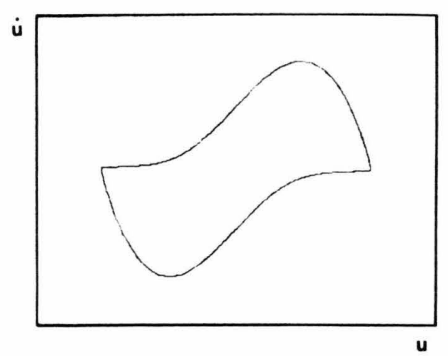

Fig. 6. Phase portrait of Van der Pol equation where $a=5$, and $n=1$.

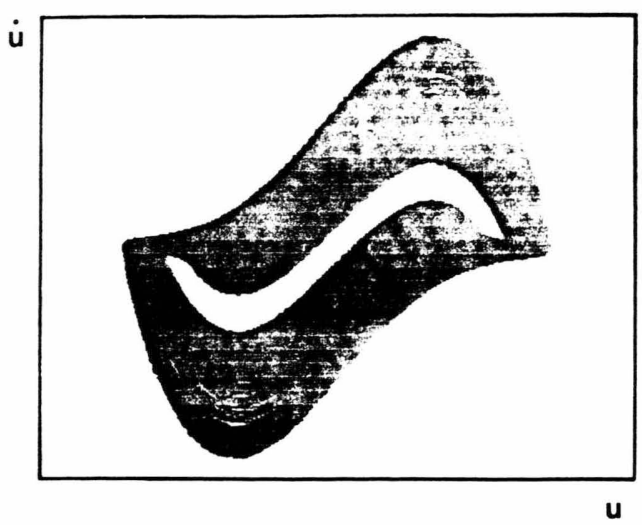

Fig. 2. Phase portrait of Van der Pol equation with external periodic force where $a=5, k=5, \Omega=2.466$, and $n=1$ (3000 cycles).

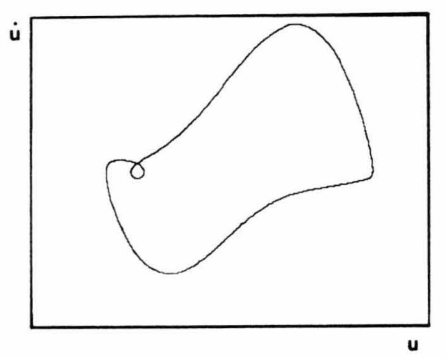

Fig. 4. Phase portrait of generalized Van der Pol equation with external periodic force where $a=5, k=5$, $\Omega=2.466$, and $n=3$.

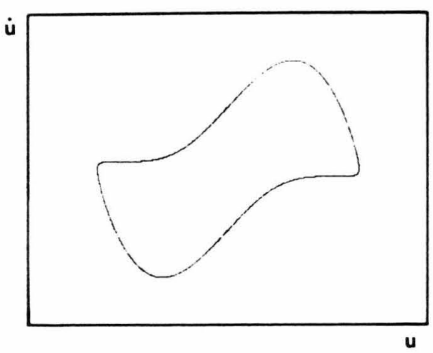

Fig. 7. Phase portrait of generalized Van der Pol equation where $a=5$, and $n=3$.

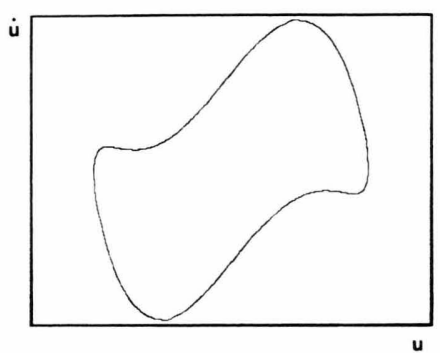

Fig. 5. Phase portrait of generalized Van der Pol equation with external periodic force where $a=5, k=5$, $\Omega=2.466$, and $n=5$.

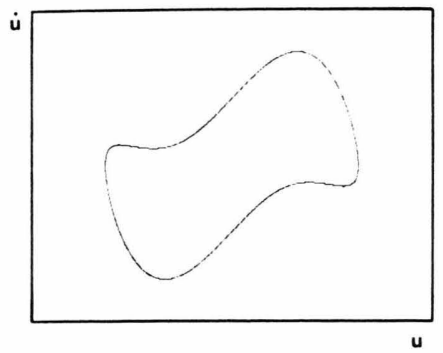

Fig. 8. Phase portrait of generalized Van der Pol equation where $a=5$, and $n=5$.
Figure 1 shows the phase portrait of (3) with $a=5$, $k=5, \Omega=2.466$ and $n=1$. The maximal onedimensional Lyapunov exponent is positive and thus chaotic behaviour is indicated. Figure 2 shows the phase portrait for a very long computer simulation. In Fig. 3 the power spectrum is depicted. The power spectrum involves the subharmonic $\Omega / 4$, together with the background "noise" (period 4-chaos).

Let us now investigate $a=5, k=5$, and $\Omega=2.466$ for $n=3,5,7$. One would expect that "introducing" an additional nonlinearity would strengthen the chaotic behaviour for these values. However, we find that 
the chaotic motion disappears for $n=3, n=5$ and $n=7$ and retains the periodic motion. Figure 4 gives the phase portrait for $n=3$ and Fig. 5 for $n=5$. We have also calculated the power spectrum, maximal one-dimensional Lyapunov exponent and Poincaré section. No chaotic motion is indicated. For $n=3$ the power spectrum involves the subharmonic $\Omega / 2$ and the frequencies $3 \Omega / 2,5 \Omega / 2,7 \Omega / 2$, etc. with decreasing amplitude. Furthermore, one finds the higher harmonics $2 \Omega, 3 \Omega, 4 \Omega$, etc. with decreasing amplitude. The subharmonic $\Omega / 2$ is related to the small loop in the limit cycle (see Figure 4). For $n=5$ no subharmonics exist and we only find higher harmonics, namely $3 \Omega, 5 \Omega$, etc. The case $n=7$ is qualitatively the same as $n=5$.

This behaviour can be explained as follows. For large $a$ (say $a=5$ ) the phase portrait of the van der Pol equation shows two "sharp corners" at $u=2$ and $u=-2$ (Figure 6). These two "sharp corners", generated by the "damping nonlinearity", are responsible for the chaotic behaviour when we introduce the external periodic force with appropriate values of $k$ and $\Omega$. The "sharp corners" disappear (the corners become

[1] N. Levinson and O. K. Smith, Duke Math. J. 9, 382 (1942).

[2] B. Van der Pol, Phil. Mag. 2, 978 (1926).

[3] U. Parlitz and W. Lauterborn, Phys. Rev. A 36, 1428 (1987). smoother and smoother) when $n=3,5,7$ for $a=5$. Figure 7 shows the phase portrait of (2) with $n=5$ and $a=5$ (Figure 8). This smoothing effect of the nonlinearity $u^{n}$ destroys the chaotic motion. The nonlinearity $u^{n}$ with $n=3,5,7$ can be derived from the potential $V(u) \propto u^{n+1}$. With increasing $n$ the "particle" becomes more and more confined.

The behaviour of the disappearing chaotic motion also arises for other values of $\Omega$ in the range $2.424 \leq \Omega \leq 2.502$, with chaotic behaviour for $n=1$.

The "sharp corners" also disappear when the value of $a$ becomes small. The stable limit cycle becomes nearly a circle for small values of $a$. For small $a$ no chaotic behaviour can be found for the van der Pol equation with an external periodic force $[4,5]$. In this case the nonlinearity $u^{n}(n=3,5,7)$ must come into play to find chaotic motion.

One of the authors (W-H S) thanks the Department of Electrophysics of the Chiao University for its hospitality during the work on this project. This work was supported by the NSC of the Republic of China and the CSIR of the Republic of South Africa.

[4] Y. Ueda and N. Akamatsu, IEEE. Trans. Circuits Syst. 28, 217 (1981).

[5] W.-H. Steeb and A. Kunick, Int. J. Non-Linear Mechanics 22, 349 (1987). 